\section{The Appendicitis Question.}

Detroit, Mich., Feb. 14, 1898.

To the Editor:-It has been a rule in my life never to go into a controversy with people, although I have sometimes sent communications to the JournaL, giving my views when I disagreed with others. My friend from Richmond, Ind., has sent a long reply to my last communication, and, after carefully reading it, $I$ have come to the conclusion that he is a nice, good fellow and a progressive physician at heart, and if we bring our views together I am sure we will perfectly agree, excepting on some minor details.

He quotes Treves and Thalamon, both of whom had limited experience with the operation of appendicitis, and whose opinions should not count alongside of such men as Robert Morris, J. B. Murphy, Joseph Price, Fowler and Deaver, men who have had hundreds of operations and seen the terrible results 'of procrastination. When a man who has operated ten, twenty or thirty times and who has had bad results, because he has not learned the minute detail of the operation, says you should not operate before the tenth day, except on those cases where a large abscess has formed, which is well walled in and which simply needs to be opened (which any tyro in surgery can do), but who, at the same time, allows a dozen other patients to die on the second, fourth, sixth or eighth day, when such men give their views they do more harm than we do who are obliged to take the other extreme.

It depends upon who operates. Often the surgeon, himself, infects the parts that are clean and the patient dies as a result of the operation, but a good abdominal surgeon, with perfect asepsis, will seldom have a patient die before rupture and general peritonitis, except as the result of some unforeseen accident. Ideas are charged to me which I never held nor expreesed. There are any number of cases where I refused to operate because I saw the patient was convalescent, but said that if they had recurrence they should have an operation performed the first day. It is easy to say you will operate at the interval, but patients, if they feel perfectly well, will not submit to an operation at the interval, for which I do not blame them, as, they say, "perhaps I will never have another attack."

The other point is diagnosis. Some men can never diagnose a case of appendicitis, or only in rare cases. My views are not expressed on cases where a diagnosis had not been made. I do not operate by guesswork. I assume, in my communication, that diagnosis is made and that, with a rare exception, it can be made in the first twenty-four hours, before rupture has taken place. I am sure the Doctor will agree with me that it is better to operate the first day, or, for that matter, the second, any time before rupture has taken place. That is all I claim. I will admit if you have a fortunate case, where the rupture and the resulting abscess is well walled in and gradually works its way outward, and in the course of ten or fourteen days points at some place near the crest of the ilium, that it is easy and not dangerous to open that and let out the pus. At the same time, before this takes place, in many cases it ruptures into the peritoneal cavity, we have general septic peritonitis, or, the absorption of septic material is so great that your patient dies (in either case) on the fifth or sixth day. So that the controversy stands really here :

My friend will accept the dictum of a general surgeon with a very limited experience in abdominal surgery, like Treves, or some young man, also with a limited experience, like Thalamon, while I prefer the views of men who have operated on a couple of hundred cases each, like Price, Deaver, Murphy, Fowler, Morris and others.

1. He and I agree that a diagnosis should first be made.

2. He and $I$ agree that a convalescent patient should not be operated upon.
3. He and I agree that patients absolutely recover without recurrence and without an operation.

4. He and I also agree that eome patiente need an operation, and if there is any difference in our views it is simply as to when is the best time.

I believe, with my limited experience, as I see only about fifty cases a year, that the greatest number of patients will be saved if they would all be promptly operated upon as soon as the diagnosis is made. So that, after all, we get so near together that there is no use splitting hairs about it, and I am perfectly convinced that Dr. Haughton is a nice, good fellow, that he means well, and what is more, $I$ am sure he is progressive and wants to arrive at the truth, otherwise, we would never have heard from him. Yours truly,

$$
\text { J. H. Carstens, M.D. }
$$

\section{The Australian Eunuchs.}

Chicago, Feb. 10, 1898.

To the Editor:-In an article on "The Oriental Eunuchs," by Dr. Edmund Andrews, in the issue of January 22, in the paragraph on "A ustralia" the author refers to the natives limiting "the increase of families by crushing the testicles of the father after his first child is born." Referring to this point the facts below are not entirely relevant, but may prove of interest in this connection.

My brother, Mr. Fred Hessert, who has for over a year been sojourning in West A ustralia, has in his letters to me described some of the customs of the barbarie natives. Owing to the barren desert condition of the country, the natives prevent a too great increase in their number by certain surgical operaations performed on the penis. When a boy reaches the age of puberty he is taken in hand by a number of old "bucks," who lead him into the "bush" and there perform on him an external urethrotomy. The opening is made with crude instruments, sharp stones, pieces of tin or old knives and the like obtained from the whites. The opening is made at the beginning of the pars pendulosa and is kept open so that eventually a fistula is produced. The object of this procedure is evident; in copulation the semen is ejected through the fistula and the possibility of impregnation reduced to a minimum. When the black desires to impregnate, he plugs up his fistula with a gum or resin obtained from a certain tree. The plug he keeps in place with a few turns of dried grass or vegetable fiber and he again becomes a potent factor.

A nother operation that is practiced is to slit up the entire urethra from the perineum anteriorly. This is more radical, and permanently prevents the possibility of impregnation.

These operations are commonly practiced in West Australia at least, and inasmuch as the letters from my brother are ex. haustive and in detail, and no mention is made of castration, I would infer that the latter procedure is practiced but very rarely in that region.

As the natives, even in the cold season, are very scantily dressed observation at this point is easy.

$$
\text { Repectfully yours, Wrldiam Hessert, M.D. }
$$

\section{How Squirrels Become Eunuchs.}

Hayneville, Ala., Feb. 10, 1898.

To the Editor:-In the Journat (Feb. 5, 1898) I read a communication from Dr. Eugene S. Talbot on the subject "Do Adult Squirrels Castrate Each Other?"

Castrated Squirrels are frequently killed in this country, and the supposition is almost universal that the operation is done through motives of jealousy in desperate combats.

This is one of the zoölogical fictions belonging in the same category with the horsehair snake.

The missing testicles have been destroyed by a parasitic worm peculiar to those organs of the sciuri family.
Yours,
C. E. Marlette, M.D. 